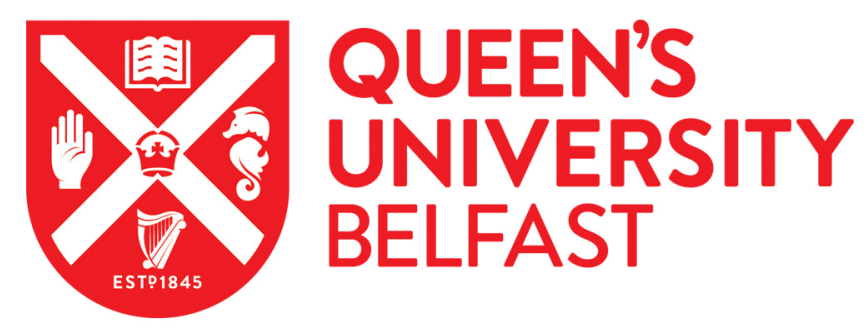

\title{
Transformer PUF: A Highly Flexible Configurable RO PUF Based on FPGA
}

Wei, Z., Cui, Y., Chen, Y., Wang, C., Gu, C., \& Liu, W. (2020). Transformer PUF: A Highly Flexible Configurable RO PUF Based on FPGA. In IEEE International Workshop on Signal Processing Systems: Proceedings (IEEE Workshop on Signal Processing Systems (SiPS): Proceedings). Institute of Electrical and Electronics Engineers Inc.. https://doi.org/10.1109/SiPS50750.2020.9195259

Published in:

IEEE International Workshop on Signal Processing Systems: Proceedings

Document Version:

Peer reviewed version

Queen's University Belfast - Research Portal:

Link to publication record in Queen's University Belfast Research Portal

Publisher rights

(c) 2020 IEEE.

This work is made available online in accordance with the publisher's policies. Please refer to any applicable terms of use of the publisher.

\section{General rights}

Copyright for the publications made accessible via the Queen's University Belfast Research Portal is retained by the author(s) and / or other copyright owners and it is a condition of accessing these publications that users recognise and abide by the legal requirements associated with these rights.

Take down policy

The Research Portal is Queen's institutional repository that provides access to Queen's research output. Every effort has been made to ensure that content in the Research Portal does not infringe any person's rights, or applicable UK laws. If you discover content in the Research Portal that you believe breaches copyright or violates any law, please contact openaccess@qub.ac.uk. 


\title{
Transformer PUF: A Highly Flexible Configurable RO PUF Based on FPGA
}

\author{
Ziwei Wei ${ }^{1}$, Yijun $\mathrm{Cui}^{1}$, Yunpeng Chen ${ }^{1}$, Chenghua Wang ${ }^{1}$, Chongyan $\mathrm{Gu}^{2}$ and Weiqiang $\mathrm{Liu}^{1}$ \\ ${ }^{1}$ College of Electronic and Information Engineering, Nanjing University of Aeronautics and Astronautics, Nanjing, China, 211106 \\ ${ }^{2}$ Centre for Secure Information Technologies(CSIT), Queen'sUniversity Belfast, Belfast, UK, BT3 9DT \\ Email: liuweiqiang@nuaa.edu.cn
}

\begin{abstract}
Physical unclonable function (PUF) is a promising security primitive for IP protection and user authentication. As there are plenty of reconfigurable resources in a field programmable gate array (FPGA), configurable ring oscillator (CRO) PUF is one of the most hardware efficient PUF designs. Previous CRO PUF designs have relatively improved the yield of challenge and response pairs (CRPs). In this paper, a highly flexible CRO PUF based on FPGA, defined as Transformer PUF, is proposed. The proposed PUF design, which has multiple reconfigurability from XOR gates and multiplexers, can be deformable between different CRO PUFs. Compared with the traditional CRO PUFs, it is more resistant to two common machine learning attack techniques, logistic regression (LR) and covariance matrix adaptation evolutionary strategies (CMA-ES), with a small sample set size. Moreover, the Transformer PUF achieves the highest hardware efficiency among CRO PUFs. The results of the experiment carried out on Xilinx Artix-7 development board demonstrate that Transformer PUF has a good uniqueness of $\mathbf{4 9 . 4 4 \%}$ and a high reliability of $98.12 \%$.

Index Terms-Physical Unclonable Function, Configurable Ring Oscillator, FPGA Implementation, Hardware Efficiency
\end{abstract}

\section{INTRODUCTION}

The information security of computing systems is very important for the Internet of Things (IoTs), from the server clusters to nodes. Traditional security primitives, e.g. advanced encryption standard (AES), are unpractical for resourceslimited or battery based devices [1]. The methods of using non-volatile memory (NVM) to store keys proved to be incapable of resisting invasive attacks [2], [3]. PUF [4] has been proposed to protect hardware security since it is energyefficient and cost-effective.

The ring oscillator (RO) PUF [5] is field programmable gate array (FPGA) friendly and can be easily deployed to FPGA-based platform [6]. Two identical ROs are chosen by challenge bits and the output frequencies are contrasted to produce a PUF response bit. Configurable RO (CRO) PUFs [7]-[11] were proposed for improving hardware efficiency of the conventional RO PUF. CRO PUFs can increase utilization rates of inverters in each $\mathrm{RO}$ by using configurable signals. In [7] and [8], multiplexers (MUXs) were used to choose inverters to form a RO. In [9], a low-cost CRO PUF design based on XOR gates was proposed. In [10], MUXs were used to decide the transmission path in a RO. In [11], tristate inverters were utilized as the configurable components and the design achieved good flexibility and low hardware cost, but it is only suitable for application specific integrated circuit (ASIC) designs and unpractical in modern FPGAs. When contrasted with the conventional RO PUF, these CRO PUF structures have relatively increased hardware efficiency and the reliability. However, these CRO PUFs suffer from machine learning attacks which can build up a mapping model between challenges and responses due to the correlationship of the configuring components. In addition, the implementations of the traditional different CRO PUF structures require redeployment on FPGA development boards, which results in a high hardware cost. To deal with these problems, a highly flexible configurable RO PUF based on FPGA is proposed in this work. More specifically, the contributions of this article are divided into four following listed points:

- A highly flexible CRO PUF, Transformer PUF, is proposed, which has multiple reconfigurability and can be configured to different CRO PUF structures. It can improve the circuit reusability and reduce hardware consumption significantly.

- Two widely studied machine learning attack techniques based on modeling, LR and CMA-ES, are utilized to assess the attack techniques resistance of Transformer PUF. Experimental results prove the proposed PUF is more resistant to both LR and CMA-ES attacks with a small sample set size when compared with previous CRO PUF designs and the improved CRO PUF structure named as MPUF.

- The proposed Transformer PUF is deployed on 12 identical Xilinx Artix-7 development board. The calculation results of PUF metrics show that it achieves a good uniqueness $(49.44 \%$, ideal $50 \%$ ) and a high reliability $(98.12 \%$, ideal $100 \%)$.

- The proposed Transformer PUF achieves the highest hardware efficiency among CRO PUF designs, indicating that Transformer PUF can generate the most CRPs when consuming the same configurable logic blocks (CLBs).

The rest part of this article will be divided into the following sections: Section II introduces the proposed Transformer PUF structure. Section III provides security analysis of Transformer PUF. Section IV calculates the metrics of Transformer PUF to verify its performance. Finally, the research work of this paper will be summarized in Section V. 


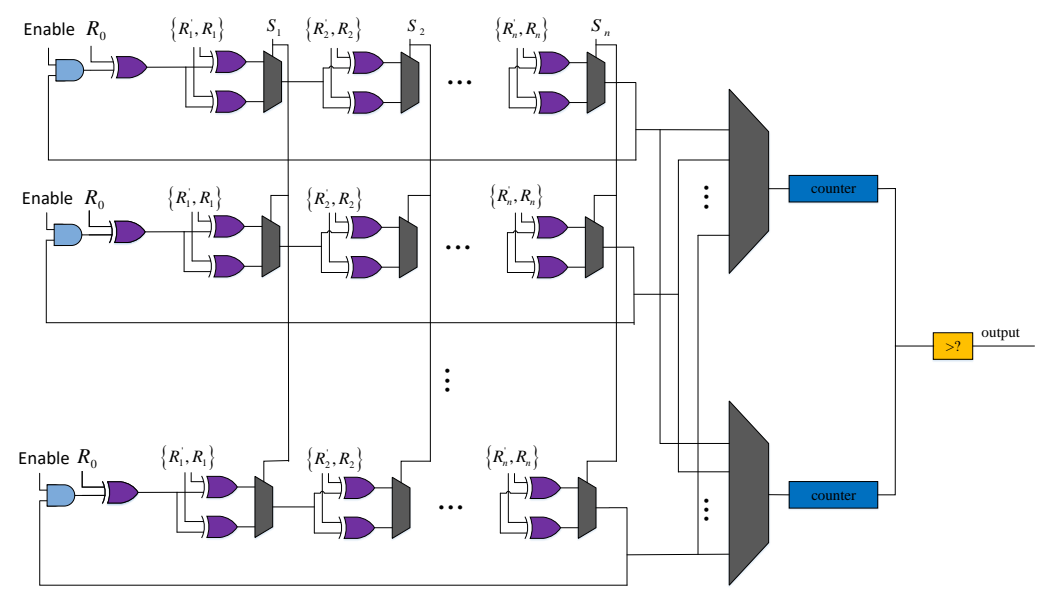

Fig. 1: The schematic of the proposed Transformer PUF design.

\section{The Proposed Transformer PUF Design}

\section{A. Basic Unit}

The schematic of the proposed Transformer PUF for generating 1-bit response is illustrated in Fig. 1. The challenge $C$ is composed of $2 n+1$ bits configuring signal for XOR gates $\left\{R_{0}, R_{1}^{\prime}, R_{1}, R_{2}^{\prime}, R_{2}, \ldots, R_{n}^{\prime}, R_{n}\right\}$ and $n$ bits selection signal for MUXs $\left\{S_{1}, S_{2}, \ldots, S_{n}\right\}$. To extract the process variations, the configurable bits should be configured identically to all configurable RO units. The proposed Transformer PUF having $n+1$ stages configurable delay units and one Enable unit is shown in Fig. 1.

The basic delay unit consists of one MUX and two XOR gates, as illustrated in Fig. 2. In the basic delay unit, one input of XOR gate is utilized as a configurable pin, denoted as $\left\{R_{i}\right\}$ or $\left\{R_{i}^{\prime}\right\}$. The other input is established a connection with the output of its pre-stage. The outputs of two XOR gates are connected to the two input pins of the MUX. The input signal of the selector pin in the MUX in each basic delay unit is denoted as $\left\{S_{i}\right\}$.

The basic unit has two dimensional reconfigurable properties. The first is based on the MUX in each delay unit, which determines the transmission path of the delay signal,

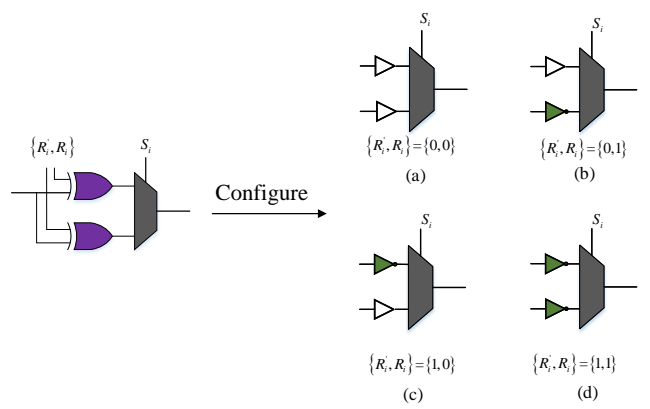

Fig. 2: The basic delay unit of the proposed Transformer PUF. similar to the designs in the conventional CRO PUF designs [7], [8]. The other configurable property relies on the XOR gates, which are the inputs of the MUX. According the XOR gate logic equivalence in Fig. 3, PUF basic delay unit can transform between different previous CRO PUF designs. For the fundamental XOR gate, if $A$ is used as a control signal, then $Q$ can be expressed as a function of $B$, that is, when $A={ }^{\prime} 0$ ', $Q=B$; when $A={ }^{\prime} 1$ ', $Q=\bar{B}$. Therefore, the operating state of the XOR gate is equivalent to two states controlled by the signal $A$ : when $A={ }^{\prime} 0$ ', the circuit can be considered as a buffer; when $A={ }^{\prime} 1$ ', it is equivalent to an inverter. Depending on the challenge signal, each basic unit can be configured to one of the four states shown in Fig. 2. When $R_{0}$ is configured as ' 0 ' and $\left\{R_{i}, R_{i}^{\prime}=(0,1)\right\}$ or $\left\{R_{i}, R_{i}^{\prime}=(1,0)\right\}$, the proposed PUF transforms to the CRO PUFs in Fig. 2 (b) or (c). When $R_{0}$ is configured as ' 0 ' and $\left\{R_{i}, R_{i}^{\prime}=(1,1)\right\}$, the proposed PUF transforms to the CRO PUF in Fig. 2 (d). When $\left\{R_{i}, R_{i}^{\prime}=(0,0)\right\}$, the basic unit works like a delay buffer. If all delay units are configured as buffers and $R_{0}$ is configured as ' 0 ', the configurable array will not oscillate. Furthermore, when $R_{0}$ is configured as ' 1 ' and odd pairs $\left\{R_{i}, R_{i}^{\prime}\right\}$ are configured as $\{1,1\}$, the circuit is converted to a configurable bistable ring (BR) PUF [12].

In general, the MUXs are utilized to select the XOR gates

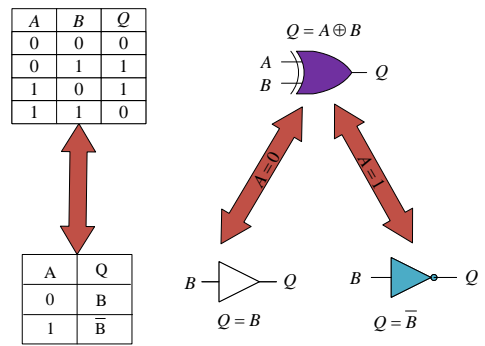

Fig. 3: The Truth table and working state of an XOR gate. 
that participate in a $\mathrm{RO}$, and the working conditions of selected XOR gates decide the working state of entire ring. For each basic unit, as long as the XOR gate in the signal transmission path selected by the MUX is configured as an inverter, the basic unit is equivalent to an inverter. The rest XOR gate that is not in the signal transmission path can be arbitrarily configured. The basic unit can be expressed as follows:

$$
F_{i}=R_{i} S_{i}+R_{i}^{\prime} \bar{S}_{i}(i=1,2, \ldots, n)
$$

where $F_{i}$ indicates the configuration of basic unit in the signal transmission path, $n$ is the number of basic unit in Transformer PUF. When $F_{i}=1$, it indicates that the corresponding basic unit is configured as an inverter, otherwise it is configured as a buffer. In order to generate effective responses, it is necessary that the total number, $\sum_{1}^{n} F(i)$, are the same odd number when $R_{0}$ is configured as ' 0 '. In this case, the circuit can oscillate and be deformed between several different CRO PUFs. The multiple reconfigurability introduced by the MUXs and the XOR gates improves circuit reusability and reduces hardware consumption.

\section{B. FPGA Implementation of Transformer PUF}

In this paper, the proposed Transformer PUF structure is deployed on Xilinx Artix-7 BASYS3 development board. To improve the hardware efficiency, the basic units of the Transformer PUF are implemented in one CLB which is FPGA basic unit. Two different SLICE types exist in one FPGA CLB, including SLICEX and SLICEL. Different SLICEs consist of different hardware resources. SLICEX has registers and look up tables (LUTs), but it lacks MUXs when compared with SLICEL. To make the best usage of the hardware resources in one CLB, this work selects the SLICEL to implement the proposed PUF structure. The LUTs in the CLB are configured as the XOR gates and AND gates in the configurable RO. The MUXF7 in the SLICEL are considered to implement the MUXs in the ROs of Transformer PUF. It avoids using fixed carry logic to configure the MUXs, which will lead to the MUX implementation taking up the entire SLICE. It also avoids occupying the limited LUT resources in the SLICE to configure MUXs because the demand for LUTs at each stage of the configurable ROs is much greater than the MUXs. The delay devices in different ROs are manually constrained by Vivado to ensure strict symmetry.

The FPGA implementation of a 3-stage proposed Transformer PUF design in one CLB is shown in Fig. 4. The orange parts represent the XOR gates and AND gates which are configured by the LUTs. The blue trapezoids are the MUXs configured by the MUXF7. The advantage of implementing the RO within one CLB is that the variations mainly come from LUTs, other than the connecting wires.

\section{SECURITy ANALYSiS}

Modeling-based machine learning techniques, which utilize the theoretical models to attack the target PUFs, have been widely utilized to imitate the CRPs mapping relationship [13][15]. Previous researches have shown that conventional CRO

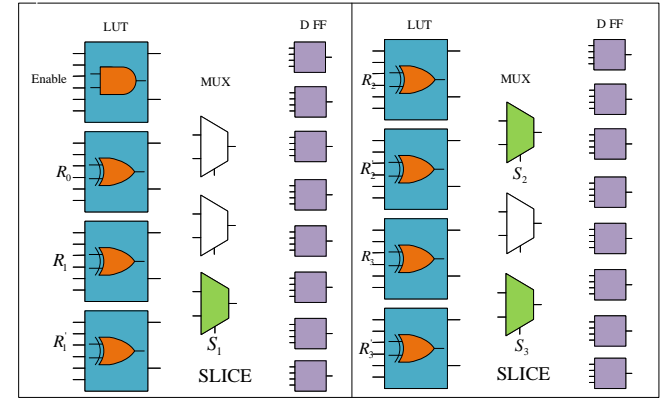

Fig. 4: The FPGA implementation of a 3-stage proposed Transformer PUF in one CLB.

PUFs are vulnerable to modeling attacks [16]. To have a comprehensive security analysis of the proposed Transformer PUF, the mathematical delay model is built and analysed.

\section{A. Delay Model of Transformer PUF}

In the basic delay unit of the Transformer PUF, the XOR gate device can be converted between an inverter and a buffer depending on the configurable signals. Hence, the delay parameters of the XOR gates can be expressed as $a_{i 1}$ or $b_{i 1}$, and $a_{i 1}^{\prime}$ or $b_{i 1}^{\prime}$, respectively, when two XOR gates of $i$-th stage in upper path RO for frequency comparison are configured as inverters or buffers by configuration signals $\left\{R_{i}\right\}$ and $\left\{R_{i}^{\prime}\right\}$. Similarly, the delay parameters of the XOR gates of $i$-th stage in lower path RO can be expressed as $a_{i 2}$ or $b_{i 2}$, and $a_{i 2}^{\prime}$ or $b_{i 2}^{\prime}$, respectively. So the delays of the XOR gates of $i$-th basic unit in upper path RO can be expressed as follows:

$$
\begin{aligned}
\Delta D_{\text {upper }}(i) & =R_{i} a_{i 1}+\left(1-R_{i}\right) b_{i 1} \\
& =R_{i}\left(a_{i 1}-b_{i 1}\right)+b_{i 1} \\
\Delta D_{\text {upper }}^{\prime}(i) & =R_{i}^{\prime} a_{i 1}^{\prime}+\left(1-R_{i}^{\prime}\right) b_{i 1}^{\prime} \\
& =R_{i}^{\prime}\left(a_{i 1}^{\prime}-b_{i 1}^{\prime}\right)+b_{i 1}^{\prime}
\end{aligned}
$$

specially $\Delta D_{\text {upper }}(0)=\Delta D_{\text {upper }}^{\prime}(0)$ since they share the same configurable XOR gate $R_{0}$.

The path delay $\triangle D_{U P P E R}(i)$ of $i$-th basic unit in upper path $\mathrm{RO}$ can be expressed as follows:

$$
\begin{aligned}
\Delta & D_{U P P E R}(i) \\
& =S_{i} \Delta_{\text {upper }}(i)+\left(1-S_{i}\right) \Delta_{\text {upper }}^{\prime}(i) \\
& =S_{i}\left(R_{i}\left(a_{i 1}-b_{i 1}\right)+b_{i 1}\right) \\
& +\left(1-S_{i}\right)\left(R_{i}^{\prime}\left(a_{i 1}^{\prime}-b_{i 1}^{\prime}\right)+b_{i 1}^{\prime}\right) \\
& =S_{i}\left(R_{i}\left(a_{i 1}-b_{i 1}\right)-R_{i}^{\prime}\left(a_{i 1}^{\prime}-b_{i 1}^{\prime}\right)+b_{i 1}-b_{i 1}^{\prime}\right) \\
& +R_{i}^{\prime}\left(a_{i 1}^{\prime}-b_{i 1}^{\prime}\right)+b_{i 1}^{\prime}
\end{aligned}
$$

where $D_{U P P E R}(0)=R_{0} a_{1}+\left(1-R_{0}\right) b_{1}$ when $i=0$.

Similarly, the path delay $\Delta D_{L O W E R}(i)$ of $i$-th basic unit in lower path RO can be expressed as follows:

$$
\begin{aligned}
& \Delta D_{\text {LOWER }}(i) \\
& \quad=S_{i} \Delta_{\text {lower }}(i)+\left(1-S_{i}\right) \Delta_{\text {lower }}^{\prime}(i) \\
& \quad=S_{i}\left(R_{i}\left(a_{i 2}-b_{i 2}\right)-R_{i}^{\prime}\left(a_{i 2}^{\prime}-b_{i 2}^{\prime}\right)+b_{i 2}-b_{i 2}^{\prime}\right) \\
& \quad+R_{i}^{\prime}\left(a_{i 2}^{\prime}-b_{i 2}^{\prime}\right)+b_{i 2}^{\prime}
\end{aligned}
$$


where $D_{L O W E R}(0)=R_{0} a_{2}+\left(1-R_{0}\right) b_{2}$ when $i=0$.

Finally, the mathematical expression of the delay difference $\Delta D(i)$ between two $i$-th basic units of the upper RO and the lower RO in Transformer PUF is as follows:

$$
\begin{aligned}
\Delta D(i) & =\Delta_{U P P E R}(i)-\Delta_{L O W E R}(i) \\
& =S_{i} R_{i}\left(a_{i 1}-b_{i 1}-a_{i 2}+b_{i 2}\right) \\
& +S_{i} R_{i}^{\prime}\left(a_{i 1}^{\prime}-b_{i 1}^{\prime}-a_{i 2}^{\prime}+b_{i 2}^{\prime}\right) \\
& +S_{i}\left(b_{i 1}-b_{i 1}^{\prime}-b_{i 2}+b_{i 2}^{\prime}\right) \\
& +R_{i}^{\prime}\left(a_{i 1}^{\prime}-b_{i 1}^{\prime}-a_{i 2}^{\prime}+b_{i 2}^{\prime}\right) \\
& +b_{i 1}^{\prime}-b_{i 2}^{\prime}
\end{aligned}
$$

where $D(0)=R_{0}\left(a_{1}-b_{1}-a_{2}+b_{2}\right)+b_{1}-b_{2}$ when $i=0$. The total delay difference $\Delta D$ is the superposition of $\Delta D(i)$. While $\Delta D(i)$ for traditional CRO PUFs $(\Delta D(i)$ of CRO PUF [7], [8], RRO PUF [10], XCRO PUF [9] can be considered the same) is proved to be $\Delta D(i)=\frac{1-C_{i}}{2}\left(\delta_{i 1}-\delta_{i 1}^{\prime}\right)+$ $\frac{1+C_{i}}{2}\left(\delta_{i 2}-\delta_{i 2}^{\prime}\right)$ in [16]. According to Eq. (6), the mathematical delay model of the proposed Transformer PUF is more complex when compared with conventional RO-based PUFs. It contains more variable combinations, which is introduced by the multiple reconfigurable properties.

\section{B. Machine Learning Attacks on Transformer PUF}

As analysed above, conventional CRO PUFs are vulnerable to modeling attack since their delay model are relatively simple. Previous MPUF attempted to increase the resistance to machine learning attack by XORing the challenges with the unknown outputs of weak PUFs in [16]. However, the improved MPUF still suffers from the machine learning attack while consuming more hardware resources. While for the Transformer PUF, the delay model in Eq.(6) demonstrates that the CRPs mapping relationship is more complicated than previous CRO PUF designs, which means it is more secure against potential attacks.

To evaluate the resistance to ML attack of classical CRO PUFs and the Transformer PUF, two common machine learning techniques, LR [13] and CMA-ES [17] are adopted. LR is one of the most efficient two-classification algorithms. In the research work of this article, the LR open source implementation [18] is used, which is available from [19]. For the non-linear PUF structure, non-linear algorithm CMAES [20] is one of the most effective attack techniques. Due to the existence of noise in the actual test environment, which is introduced by the variations from the supply voltage and the environmental temperature, a Gaussian variable of norm $\left(\mu=0, \sigma_{\text {noise }}=0.5\right)$ is added to all delay differences $\Delta D(i)$ of PUFs in machine learning training. To have a more comprehensive analysis of the proposed Transformer PUF, basic units which contain different stages of the XOR gates (denoted as Transformer PUF based on 2 XOR gates, 4 XOR gates and 8 XOR gates) are constructed in this evaluation.

The prediction rates under different modeling attacks for conventional CRO PUF and Transformer PUF are shown in Fig. 5 and Fig. 6. As illustrated in Fig. 5, the prediction rate

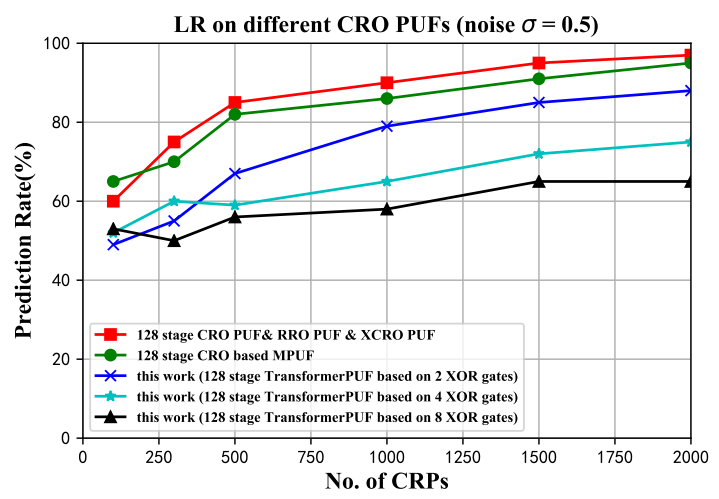

Fig. 5: LR result on different CRO PUFs.

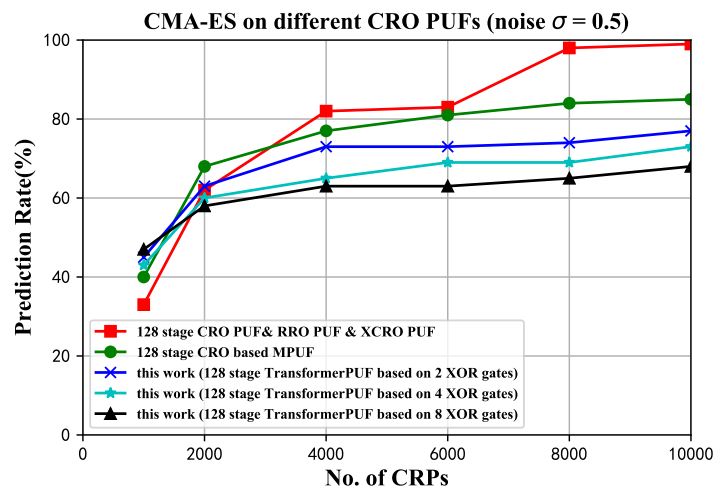

Fig. 6: CMA-ES result on different CRO PUFs.

of the proposed PUF is always significantly lower than that of traditional CRO PUFs and the improved MPUF structure with the same training sample set size. However, the prediction rate under LR attack of the improved MPUF structure is only slightly lower than that of traditional CRO PUFs. For a 128 stage Transformer PUF based on 8 XOR gates, the prediction rate is around $60 \%$ with 2000 CRPs while traditional CRO PUFs and the improved MPUF structure have exceeded $90 \%$. Fig. 6 gives the prediction rates of different CRO PUFs under CMA-ES attack. The prediction rates of all PUF designs increase with the rise of the training dataset size. However, the response prediction effect of the Transformer PUF gets worse when the quantity of reconfigurable XOR gates increases with the same train set size. Therefore, Transformer PUF has better resistance to the CMA-ES attack when compared with existing CRO PUF designs. Furthermore, it has been proved that the server can return fake responses when modeling attack is detected, which results in attackers not being able to obtain infinity actual CRPs [21]. The Transformer PUF can achieve a good balance between hardware consumption and resistance to modeling attack, especially for FPGA based platform.

\section{PUf Performance EVAluation}

In order to calculate the metrics of Transformer PUF, including reliability and uniqueness, this work deploys Transformer 
PUF on 12 identical Xilinx Artix-7 development boards and tests these PUFs in a range of $0^{\circ} \mathrm{C} \sim 70^{\circ} \mathrm{C}$.

\section{A. Uniqueness}

The uniqueness means the jump degree of response bits of the PUF chips with the same structure to the same challenge [22], its mathematical expression is as follows:

$$
\text { uniqueness }=\frac{2}{d(d-1)} \sum_{i=1}^{d-1} \sum_{j=i+1}^{d} \frac{H D\left(R_{i}, R_{j}\right)}{n} \times 100 \%
$$

where $d$ is the quantity of PUF devices with the same structure; $R_{i}$ and $R_{j}$ represent the generated responses of different PUFs with the same structure under identical external environment; $n$ is the PUF response digits. According to Fig. 7, the uniqueness value can be calculated as $49.44 \%$.

\section{B. Reliability}

Reliability reflects the ability of the PUF chip to maintain the response to the same challenge under different voltage and temperature [22]. Its mathematical expression is as follows:

$$
\text { reliability }=1-\frac{1}{m} \sum_{j=1}^{m} \frac{H D\left(R, R_{j}\right)}{n} \times 100 \%
$$

where $m$ is the quantity of tests; $R$ is the corresponding response obtained at normal atmospheric temperature and standard voltage; $R_{j}$ is the response obtained in $j$-th different environments. Fig. 8 shows the intra-chip HD of Transformer PUF and its mean value is $1.88 \%$. Thus, the reliability of Transformer PUF is $98.12 \%$.

The comparison of different CRO PUFs' metrics is illustrated in Table I. For uniqueness, Transformer PUF is better than most CRO PUFs, only slightly lower than RRO PUF. Because the Transformer PUF structure is relatively more complex, the reliability is relatively lower, but it is also close to $100 \%$. In summary, Transformer PUF has better uniqueness and reliability performance among CRO PUFs.

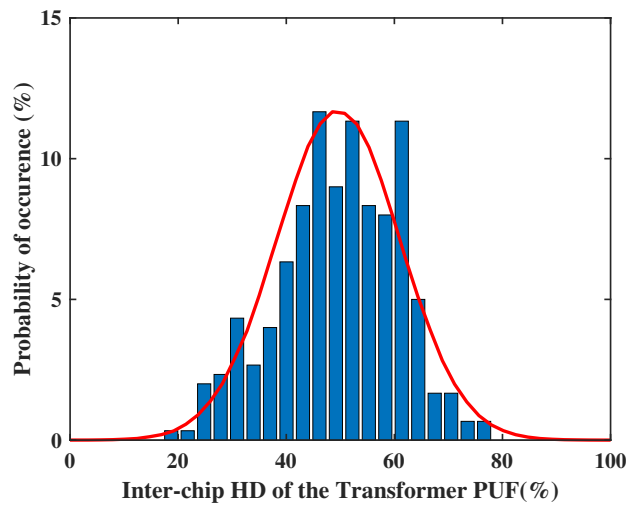

Fig. 7: The inter-chip HD of the Transformer PUF.

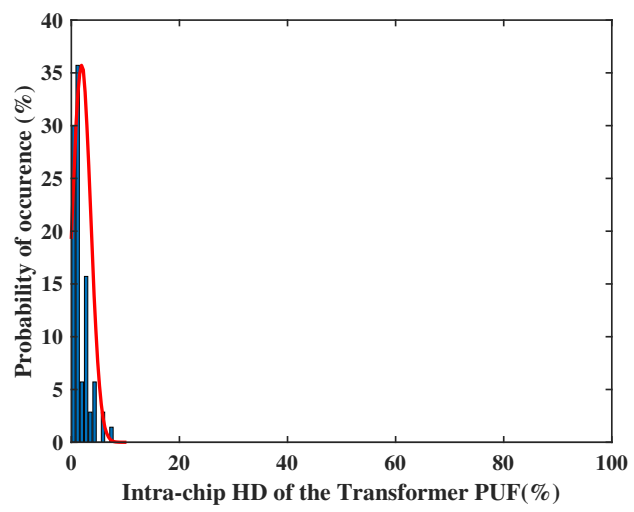

Fig. 8: The intra-chip HD of the Transformer PUF.

\section{TABLE I: THE PERFORMANCE COMPARISON}

\begin{tabular}{lll} 
CRO PUFs & Uniqueness & Reliability \\
\hline RO PUF [5] & $46.1 \%$ & $99.52 \%$ \\
CRO PUF [7] & $47.31 \%$ & $99.14 \%$ \\
RRO PUF [10] & $49.97 \%$ & $97.40 \%$ \\
XCRO PUF [9] & $48.85 \%$ & $97.72 \%$ \\
Proposed Transformer PUF & $49.44 \%$ & $98.12 \%$ \\
\hline
\end{tabular}

\section{Hardware Efficiency}

Hardware efficiency (HE) can be evaluated by the quantity of CLBs needed to generate one PUF response bit in [9]. The $\mathrm{HE}$ of the PUF can be expressed as:

$$
H E=\frac{N_{C L B}}{N_{R b i t}}
$$

where $N_{C L B}$ represents the quantity of CLB and $N_{\text {Rbit }}$ represents the response digits. Resource consumption is determined by the quantity of CLBs occupied in the FPGA hardware implementation. Assuming that one CLB is only used to implement one kind of RO, when the CRO PUF has $n$ ROs, it can generate one $C_{N}^{2}$-bit response due to frequency comparison. In the evaluation, a 3-stage Transformer PUF can be deployed into one CLB. According the analysis in Section II, there are 4 working modes for each delay unit. Moreover, the remaining LUT in the CLB can be configured as the configurable XOR gate $R_{0}$. So in total, there are $\frac{4^{3} \times 2}{2}=64$ valid configuration combinations for circuit oscillation. Thus, the HE of the Transformer PUF can be calculated as follows:

$$
H E_{\text {Transformer PUF }}=\frac{2 n}{64 n(n-1)}
$$

HE calculation results of other CRO PUFs are as follows:

$$
\begin{aligned}
H E_{R O[5]} & =\frac{2 n}{n(n-1)} \\
H E_{C R O[8]} & =\frac{2 n}{4 n(n-1)}
\end{aligned}
$$




\section{REFERENCES}

$$
H E_{C R O[7]}=H E_{R R O[10]}=\frac{2 n}{8 n(n-1)}
$$

For CRO based on MPUF, there are only two configuration possibilities in one CLB since one-stage MPUF requires 6 LUTs, 1 MUX, and 2 D-FFs to configure the CRO, ENABLE side and PicoPUF. So the HE of the MPUF based on CRO can be expressed as follows:

$$
H E_{M P U F \text { based on } C R O}=\frac{2 n}{2 n(n-1)}
$$

The comparison of the HEs between Transformer PUF and conventional CRO PUFs is shown in the Fig. 9. According to the HE in Eq. (10) and the result in Fig. 9, in order to generate the same numbers of CRPs, the CLB resource consumed of the proposed PUF is only $1 / 64$ of the RO PUF, $1 / 16$ of the CRO PUF [8], 1/8 of the CRO PUF [7] and $1 / 8$ of the RRO PUF. From Fig. 9, it is clear that the transformer PUF has the highest hardware efficiency among existing CRO PUFs.

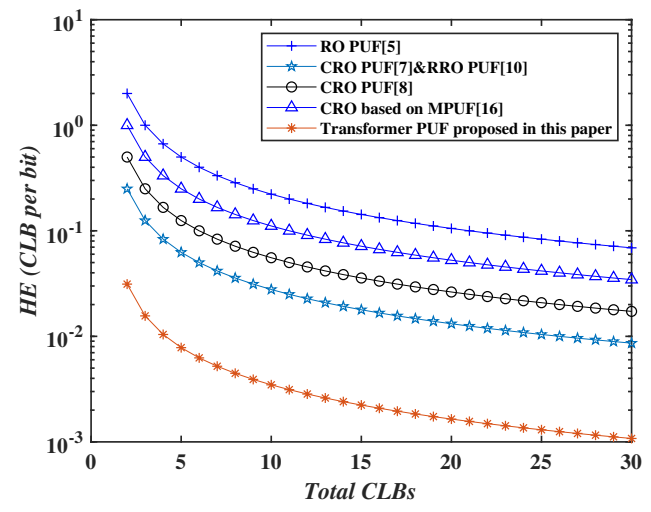

Fig. 9: HE comparison with CRO PUFs.

\section{CONCLUSION}

A highly flexible configurable RO PUF based on FPGA is proposed in this paper. It can transform between different CRO PUF designs corresponding to the configurable signals. There is no need to redeploy FPGA development boards for different CRO PUFs, which improves circuit reusability and reduces hardware consumption. Due to the multiple configurable primitives introduced by XOR gates and MUXs, the complexity of the CRPs mapping relationship increases, making it more resistant to machine learning attacks with a small sample set of CRPs. Moreover, the Transformer PUF achieves the highest hardware efficiency when compared with previous CRO PUFs and the improved MPUF structure. Experiment results also demonstrate that the Transformer PUF has an excellent uniqueness as well as a very high reliability.
[1] J. Zhang, G. Qu, Y. Lv, and Q. Zhou, "A Survey on Silicon PUFs and Recent Advances in Ring Oscillator PUFs," Journal of computer science and technology, vol. 29, no. 4, pp. 664-678, 2014.

[2] S. Guo, X. Zhao, F. Zhang, T. Wang, Z. J. Shi, F.-X. Standaert, and C. Ma, "Exploiting the Incomplete Diffusion Feature: A Specialized Analytical Side-channel Attack Against the AES and Its Application to Microcontroller Implementations," IEEE Transactions on Information Forensics and Security, vol. 9, no. 6, pp. 999-1014, 2014.

[3] M. S. E. Mohamed, S. Bulygin, M. Zohner, A. Heuser, M. Walter, and J. Buchmann, "Improved Algebraic Side-channel Attack on AES," in IEEE International Symposium on Hardware-Oriented Security and Trust, pp. 146-151, 2012.

[4] G. Hammouri and B. Sunar, "A Tamper-proof and Lightweight Authentication Scheme," Pervasive and mobile computing, vol. 4, no. 6 , pp. 807-818, 2008.

[5] G. E. Suh and S. Devadas, "Physical Unclonable Functions for Device Authentication and Secret Key Generation," in Proc. 44th ACM Annual Design Automation Conference, pp. 9-14, 2007.

[6] P. Sedcole and P. Y. Cheung, "Within-die Delay Variability in $90 \mathrm{~nm}$ FPGAs and Beyond," in IEEE International Conference on Field Programmable Technology, pp. 97-104, 2006.

[7] A. Maiti and P. Schaumont, "Improved Ring Oscillator PUF: An FPGAfriendly Secure Primitive," Journal of cryptology, vol. 24, no. 2, pp. 375397, 2011.

[8] M. Gao, K. Lai, and G. Qu, "A Highly Flexible Ring Oscillator PUF," in Proc. 51st ACM Annual Design Automation Conference, pp. 1-6, 2014

[9] L. Zhang, C. Wang, W. Liu, M. O'Neill, and F. Lombardi, "XOR Gate Based Low-cost Configurable RO PUF," in Proc. IEEE International Symposium on Circuits and Systems (ISCAS), pp. 1-4, 2017.

[10] Y. Cui, C. Wang, W. Liu, Y. Yu, M. O'Neill, and F. Lombardi, "Lowcost Configurable Ring Oscillator PUF with Improved Uniqueness," in Proc. IEEE International Symposium on Circuits and Systems (ISCAS), pp. 558-561, 2016.

[11] Y. Cui, C. Gu, C. Wang, M. O'Neill, and W. Liu, "Ultra-Lightweight and Reconfigurable Tristate Inverter Based Physical Unclonable Function Design," IEEE Access, vol. 6, pp. 28478-28487, 2018.

[12] Q. Chen, G. Csaba, P. Lugli, U. Schlichtmann, and U. Rührmair, "The Bistable Ring PUF: A New Architecture for Strong Physical Unclonable Functions," in Proc. IEEE International Symposium on HardwareOriented Security and Trust, pp. 134-141, June 2011.

[13] U. Rührmair, F. Sehnke, J. Sölter, G. Dror, S. Devadas, and J. Schmidhuber, "Modeling Attacks on Physical Unclonable Functions," in Proc. 17th ACM Conference on Computer and Communications Security (CCS), pp. 237-249, ACM, 2010.

[14] J. Delvaux, "Machine-learning Attacks on PolyPUFs, OB-PUFs, RPUFs, LHS-PUFs, and PUF-FSMs," IEEE Transactions on Information Forensics and Security, vol. 14, no. 8, pp. 2043-2058, 2019.

[15] H. Su and J. Zhang, "Machine Learning Attacks on Voltage Overscaling-based Lightweight Authentication," in Asian Hardware Oriented Security and Trust Symposium (AsianHOST), pp. 50-55, 2018.

[16] J. Miskelly, C. Gu, Q. Ma, Y. Cui, W. Liu, and M. O’Neill, "Modelling Attack Analysis of Configurable Ring Oscillator (CRO) PUF Designs," in Proc. IEEE 23rd International Conference on Digital Signal Processing (DSP), pp. 1-5, Nov 2018.

[17] N. Hansen, "The CMA Evolution Strategy: A Comparing Review," in Towards a new evolutionary computation, pp. 75-102, Springer, 2006.

[18] U. Rührmair, F. Sehnke, J. Sölter, G. Dror, S. Devadas, and J. Schmidhuber, "Modeling attacks on physical unclonable functions," in Proceedings of the 17th ACM conference on Computer and communications security, pp. 237-249, 2010.

[19] Tum, "Physical cryptography project," tech. rep., Accessed on : Jun 07, 2018.

[20] G. T. Becker, "The gap between promise and reality: On the insecurity of XOR arbiter PUFs," in in Proc. International Workshop on Cryptographic Hardware and Embedded Systems, pp. 535-555, Springer, 2015.

[21] C. Gu, C. H. Chang, W. Liu, S. Yu, Q. Ma, and M. O'neill, "A modeling attack resistant deception technique for securing puf based authentication," in 2019 Asian Hardware Oriented Security and Trust Symposium (AsianHOST), pp. 1-6, 2019.

[22] C.-H. Chang, Y. Zheng, and L. Zhang, "A Retrospective and A Look Forward: Fifteen Years of Physical Unclonable Function Advancement," IEEE Circuits and Systems Magazine, vol. 17, no. 3, pp. 32-62, 2017. 